\title{
A tool for analysis of the influence of the Earth surface soil layer temperature on the inhomogeneity of grain crops development by the Earth remote sensing data
}

\author{
Ruslan V. Brezhnev ${ }^{1}$, Yuriy A. Maglinets ${ }^{1}$, Ksenia V. Raevich ${ }^{1}$ and \\ Vardui G. Margaryan ${ }^{2}$ \\ ${ }^{1}$ Institute of Space and Information Technology of Siberian Federal University, Krasnoyarsk, Russia \\ ${ }^{2}$ Yerevan State University, Yerevan, Armenia
}

\begin{abstract}
The work is devoted to the analysis of the influence of the earth surface temperature on the inhomogeneity of the agricultural crops development. The aim of the work is to expand the object-relational model for describing the inhomogeneous spatial structure of a spatial object by including surface temperature as one of the key features that allow determining the cause of vegetation heterogeneity, along with relief features, differences in the soil chemical composition and other significant characteristics.

Experimental studies are carried out at sites located in Sukhobuzimsky district of Krasnoyarsk Territory, for which agricultural crops (grains) and the their sowing dates are known a priori, which allows stating any facts of the vegetation development deviation from the normative trajectory with reference to the sequence and timing norms of phenological phase changing.

Landsat-8 OLI (Operational Land Imager) TIRS (Thermal Infrared Sensor) data are used as initial data for temperature measurements. Objects of research are presented in the form of a polygon map in SHP format.

The temperature values are calculated using the algorithm for estimating the earth temperature developed by Weng Q., Lu D. and Schubring J. The surface reflectance values are the NDVI vegetation index values also obtained from the Landsat- 8 OLI data that underwent atmospheric correction by the DOS method.

The research results are implemented in the form of a software module and integrated into the Earth remote monitoring (ERM) system of SFU Space and Information Technologies Institute (SITI). The results are used within the concept of object-oriented monitoring of spatial objects developed by the team of authors, and represent index images of the surface temperature of objects, as well as vector schematic maps.
\end{abstract}

\section{Keywords}

Earth remote sensing, Earth surface temperature, spatial object monitoring, agricultural crops, inhomogeneity, inhomogeneity structure of spatial object, dynamic model of spatial object.

\section{Introduction}

As an agromonitoring task, quantitative assessment of the state of crops becomes more complicated as the dispersion of the phytocenosis structure appears in a separate field contour due to inhomogeneity of its development. Uniform development of vegetation depends on a number of

SDM-2021: All-Russian conference, August 24-27, 2021, Novosibirsk, Russia

$\bigoplus$ brejnev.ruslan@gmail.com (R.V. Brezhnev)

(c) (1) $\odot 2021$ Copyright for this paper by its authors. Use permitted under Creative Commons License Attribution 4.0 International (CC BY 4.0).

[DUR Workshop Proceedings (CEUR-WS.org) 
natural climatic conditions, such as chemical composition of soils, soil and air temperature and humidity, wind speed and direction, level of environmental radiation, intensity of precipitation, etc. In addition, the homogeneity is influenced by anthropogenic conditions, which change the natural conditions either purposefully, thereby affecting the course of vegetation development, like agro-technological measures, or indirectly, through the influence of industrial facilities, energy, transport, pipeline communications, etc.

From the standpoint of aerospace monitoring of the earth surface, uniformity (or homogeneity) of vegetation development is manifested in the same or close values of spectral or textural properties measured at many points of the image fragment corresponding to the agricultural contour. Such state of a spatial object, recorded at the k moment of time, can be considered a reference if it corresponds to a phenological phase inherent to a given time interval in the totality of the properties analyzed.

However, long-term remote and field observations of the grain crops development in Sukhobuzimsky district of Krasnoyarsk Territory have shown that only $30 \%$ of the objects remained homogeneous throughout the growing season. Vegetation within the contours of most fields was ahead and lagging behind the normative growth rates, thus requiring to consider the field contour as an object with an inhomogeneous spatial structure. Therefore, the values of spectral or textural features for such objects are distributed unevenly and form separate homogeneous areas within the object contour.

To assess the state of vegetation in inhomogeneity conditions, it is necessary to have the means to localize the inhomogeneous structure, on the one hand, a model that would allow adequate description of the dependence of each inhomogeneous area within a spatial object on environmental conditions. Previously, the authors developed the $M_{O}$ model [1], which helps represent the inhomogeneous structure of some $j$ spatial object $O_{j}$ as its decomposition into its component parts (1). Each structural element is the $i$ inhomogeneous region of the object $O_{j}-w_{i}, i=\overline{1, N}$. In this case, each $w_{i}$ region is described by a set of features that are compared in the form of an object-relational structure, which make it possible to determine the current state $f_{A}^{i}$ for $w_{i}$ :

$$
M_{O j}=\left\langle I D,\left\{P_{\Pi j}^{i}\right\}, N S_{j}^{i}, N P_{j}^{i}, T_{j}^{i}, \bar{N}_{j}^{i}, T m, k_{j}^{i}, e_{j}^{i}, \phi_{j}^{i}, f_{A j}^{i}\right\rangle,
$$

where $I D$ is identifier of object $O_{j}$ (field number), $\left\{P_{\Pi j}^{i}\right\}$ is a set of coordinates of region $w_{i}$, $N S_{j}^{i}$ is area, $N P_{j}^{i}$ is perimeter, $T_{j}^{i}$ is thickness, $\bar{N}_{j}^{i}$ is mean NDVI value, $T m$ is time of image acquisition and localization of the object structure.

Thus, the object-relational model (2) includes spectral, metric features and a number of semantic descriptions associated with specific values or ranges of values of the listed features. The model enables to describe the shape, size and position of an object in space, and to ascertain a subset of the actual states $\left(f_{A}^{i}\right)$ of the object regions. However, this model does not contain a block describing probable causes of the inhomogeneity, which would be important for timely decision-making on the impact on the regions that are lagging behind the norm. Among the main reasons for the occurrence of the inhomogeneity are non-uniformity of the soil chemical composition, as well as its moisture content $\tilde{\nu}_{\text {soil }}$ and temperature $t_{\text {soil }}$ [2].

There are a number of objective restrictions that preclude determining the soil chemical composition and surface moisture using Earth remote sensing (ERS) methods with acceptable 
accuracy. In case with the chemical composition, this is due to the lack of suitable open ERS data, and the lack of up-to-date data from agrochemical services or access to the same, while detailed determination of moisture is difficult due to high generalization of information in one pixel of open low resolution data (for example, MODIS or MetOp).

Thus, this work addresses determination of the earth surface temperature as a feature that expands the model for describing the inhomogeneous structure of an object and as a factor influencing the occurrence of vegetation inhomogeneity.

\section{Modeling the temperature distribution over the surface of a spatial object with an inhomogeneous dynamically changing structure}

Landsat-8 OLI (Operational Land Imager) TIRS (Thermal Infrared Sensor) data is used as a baseline for temperature measurements. The $10^{\text {th }}$ channel of TIRS1 $(10.6-11.19 \mu \mathrm{m})$ was taken as a basis. The objects of research are identified on the territory of Sukhobuzimsky district of Krasnoyarsk Territory and are presented in the Earth remote monitoring system (ERMS ISIT) in the form of vector polygonal objects. A plain object without significant elevation differences, containing forest outliers, is considered as an example. The coordinates of the object center are $56.4261 \mathrm{~N} 92.9590 \mathrm{E}$.

During the growing season of 2018, the state of vegetation at the site was monitored according to Landsat-8 and Sentinel-2 data, as a result of which schematic maps of inhomogeneity were obtained (Table 1) by reference to the homogeneity criteria (2) obtained through identifying the correlation dependence of NDVI spectral index values, field spectrometric measurements and phenological phases of grain crops development [3]:

$$
s(x, y)=\left\{\begin{array}{lll}
\lambda_{0} & \text { at } & -1 \leq f(x, y) \leq 0.025 \\
\lambda_{1} & \text { at } & 0.025 \leq f(x, y) \leq 0.26 \\
\lambda_{2} & \text { at } & 0.26 \leq f(x, y) \leq 0.43 \\
\lambda_{3} & \text { at } & 0.43 \leq f(x, y) \leq 0.57 \\
\lambda_{4} & \text { at } & 0.57 \leq f(x, y) \leq 0.65 \\
\lambda_{5} & \text { at } & 0.65 \leq f(x, y) \leq 0.72 \\
\lambda_{6} & \text { at } & 0.72 \leq f(x, y) \leq 0.85
\end{array}\right.
$$

where $f(x, y)$ is NDVI value, $s(x, y)$ - segmented image, $\lambda$ are $w_{i}$ region labels interpreting the region status. Thus, $\lambda_{0}$ is a background, $\lambda_{1}$ is dragging or ploughing, $\lambda_{2}$ is sowing or harvesting, $\lambda_{3}$ is sprouting and upgrowing, yellow ripeness, $\lambda_{4}$ is tillering, $\lambda_{5}$ is booting, $\lambda_{6}$ is earing and blooming.

The NDVI values are calculated with regard to atmospheric correction performed by the DOS (Dark-Object Subtraction) method:

$$
P_{\lambda}=\frac{\pi \cdot d^{2} \cdot Q_{\text {cal }}-L_{\text {haze }}}{E_{\text {sun }} \cdot \cos ^{2} Q},
$$

where $d$ is the value of the Earth to Sun distance at the shooting point during the survey; $Q$ is 
Table 1

Results of the study of the vegetation intensity dependence on the surface temperature.

\begin{tabular}{|c|c|c|c|c|c|}
\hline Date & $\begin{array}{c}\text { Surface } \\
\text { temperature, }{ }^{\circ} \mathrm{C}\end{array}$ & $\begin{array}{l}\text { Vegetation } \\
\text { intensity }\end{array}$ & $\begin{array}{l}\text { Temperature } \\
\text { distribution } \\
\text { over the object } \\
\text { surface, }{ }^{\circ} \mathrm{C}\end{array}$ & $\begin{array}{c}\text { Air temperature } \\
\text { according to the } \\
\text { meteorological station } \\
\text { at the shooting time, }{ }^{\circ} \mathrm{C}\end{array}$ & $\begin{array}{l}\text { Shooting } \\
\text { time, } \\
\text { GMT+7 }\end{array}$ \\
\hline 2018-04-20 & & & & $2.8-6.8$ & $11: 52$ \\
\hline 2018-05-31 & & & & $23.7-27.1$ & $11: 45$ \\
\hline 2018-06-07 & & & & $21.3-23.6$ & $11: 51$ \\
\hline 2018-06-23 & & & & $27.5-30.0$ & $11: 51$ \\
\hline 2018-07-02 & & & & $27.7-31.4$ & $11: 45$ \\
\hline 2018-07-09 & & & & $18.3-24.6$ & $11: 51$ \\
\hline 2018-07-25 & & & & $18.3-21.9$ & $11: 52$ \\
\hline 2018-08-10 & & 2 & & $23.2-29.3$ & $11: 52$ \\
\hline $2108-08-26$ & & & & $20.9-26.9$ & $11: 52$ \\
\hline 2018-09-20 & & 1 & 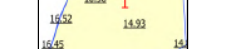 & $4.8-14.5$ & $11: 46$ \\
\hline 2018-10-13 & & & 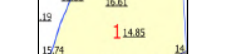 & $6.9-17.8$ & $11: 52$ \\
\hline
\end{tabular}




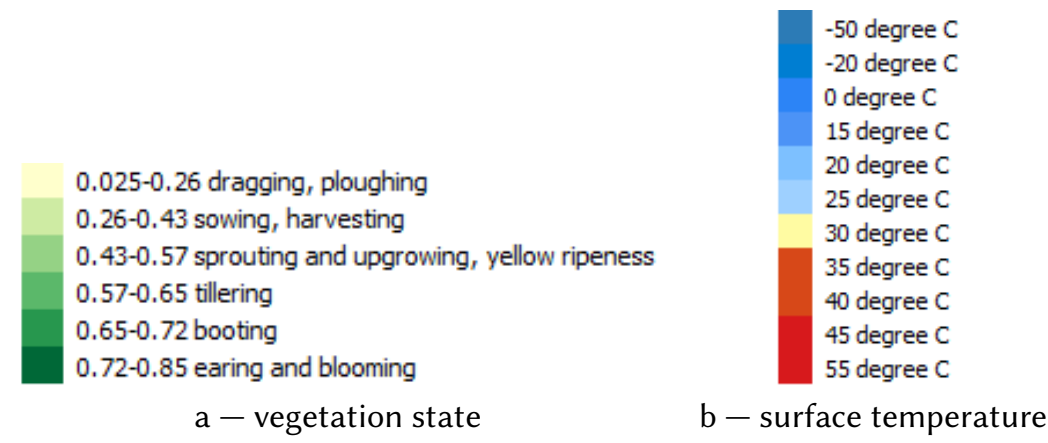

Figure 1: Legend.

zenith distance value (taken from metafiles); $E_{\text {sun }}$ is solar spectral radiation coefficient; $Q_{c} a l$ is $D N(i, j)$ pixel value of the $k$ image channel.

The method is based on subtracting the pixel $(D N)$ spectral brightness value, which corresponds to a completely black object or region in the image, from the entire image. The idea of the method is based on finding the $D N$ value of an absolutely black object, the total spectral pixel brightness of which should not exceed 0.01 , i.e. no more than $1 \%$ of the total spectral brightness of all image pixels.

The temperature values are calculated using the Earth surface temperature estimation algorithm developed by Weng Q., Lu D. and Schubring J. [4]:

$$
L S T=\left[\frac{T B}{1+\left(\frac{\lambda \cdot T B}{c_{2}}\right) \cdot \ln (N D V I)}\right]-T,
$$

where $L S T$ is the surface temperature in degrees Celsius; $T B$ is the brightness temperature of the atmospheric surface $(\mathrm{K}) ; T$ is a constant equal to one degree Kelvin $(273.15 \mathrm{~K}) ; \lambda$ is the wavelength of the emitted radiation for the tenth TIRS1 Landsat- 8 channel equal to 10.8 ; and $c_{2}$ is a constant equal to $14388 \mu \mathrm{m} \cdot \mathrm{K}$, obtained from the expression:

$$
c_{2}=h \cdot c / s,
$$

where $h$ is Planck's constant, $c$ is light velocity, and $s$ is Boltzmann constant.

Data of the surface types classification, the constant or the vegetation indices values can be used as the values of the surface emissivity in the formula (4) [4]. In this work, the NDVI values, also obtained from the Landsat- 8 OLI data, are used as the emissivity values subject to atmospheric correction.

\section{Results and discussion}

A number of experimental measurements of surface temperature were carried out during the growing season from April 20 to October 13, 2018, when the state of grain crops vegetation was monitored, in particular, at the selected object. Each time point Tm for determining the 
state of vegetation corresponds to a temperature map presented in the form of multi-temporal fragments of a pseudo-color temperature image (Table 1, column 2 - "Surface temperature") and schematic maps of vegetation states (Table 1, column 3 - "Intensity of vegetation"), designated by numbers from 0 to 6 in accordance with (2). Interpretation of the vegetation state values and temperature is presented in the legend (Fig. $1 \mathrm{a}, \mathrm{b}$ ).

For clarity, column 4 shows the temperature distribution over the observed object surface, while column 5 shows the air temperature readings obtained from the nearest meteorological station located $21 \mathrm{~km}$ to the northeast (Sukhobuzimskoye village) for the time interval closest to the shooting time (from 10:00 to 13:00) as shown in column 6 .

When summarizing the surface temperature readings in relation to the time points $T m_{k}$ ( $k=\overline{1, K}, K=11$ ) of the spatial object localization and its structure, it should be noted that the surface heating does not always correlate with the air temperature. Thus, at lower values of air temperature, higher values of surface temperature are observed and vice versa. This is due to the direct effect of solar radiation, which means that in a relatively transparent atmosphere, the surface heats up more than air and gives off thermal energy more slowly.

High intensity of vegetation was observed in those regions of the object where the surface temperature indicators during the growing season were predominantly in the range from 15 to $31^{\circ} \mathrm{C}$. As a rule, at the moments of localization such regions were in a normative state in accordance with the current phenological phases. Low intensity of vegetation was observed in regions with high temperatures - above $31^{\circ} \mathrm{C}$. The state of vegetation in such regions lagged behind the norm. There was no significant advance in the development of vegetation. In most cases, a slight advance in time corresponds to an earlier transition from the current phenological phase to the next one.

It is also noted that areas with open soil are warming up more than those covered with vegetation, and the denser the vegetation, the lower the temperature. Thus, high density of vegetation has a beneficial effect on the intensity of growth.

Figure 2 shows a graph summarizing the behavior of vegetation in the dominant region of the spatial object under study, when the surface temperature changes.

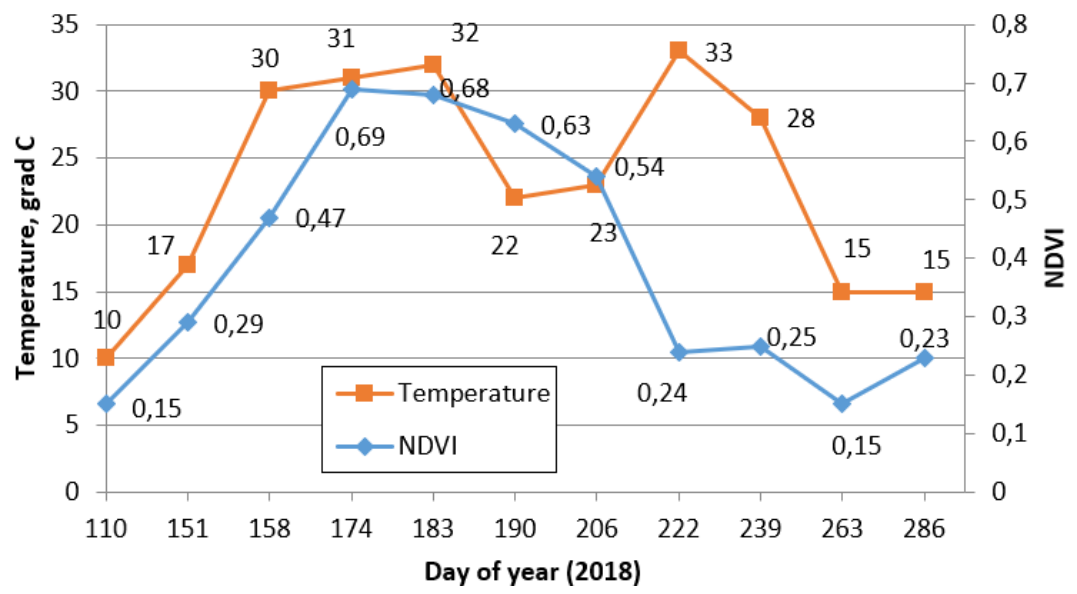

Figure 2: Intensity of vegetation of the object dominant region depending on the surface temperature. 
According to the NDVI values, the graph describes the normal trajectory of vegetation development, corresponding to the timing norms of the phenological phase changes.

\section{Conclusion}

For the model (2) of a spatial object with an inhomogeneous dynamically changing spatial structure, a block for estimating the surface temperature was developed, which made it possible to expand the description of wi regions of the object, including one of the key features that help partially analyze the causes of vegetation inhomogeneity. The block is implemented as an independent software component, has passed practical testing and is built into the Earth Remote Sensing Monitoring System of Institute of Space and Information Technology.

Further development of the model involves inclusion of the moisture parameter $\tilde{\nu}_{\text {soil }}$, which can be reconstructed from the surface temperature $t_{\text {soil }}$ and the NDVI values using the triangle or parallelepiped method [5]. It is also worth considering that $\tilde{\nu}_{\text {soil }}$ and $t_{\text {soil }}$ are very dependent on the object surface relief, on the location proximity to water bodies, forest belts or other objects shading part of the surface, therefore, for an objective assessment of inhomogeneity occurrence, the model should include difference in heights, relief and other parameters.

\section{References}

[1] Brezhnev R.V., Maglinets Yu.A. The dynamic model of agricultural land structure on the space images in the precision agriculture tasks // E3S Web of Conferences. 2019 Vol. 75. Article Number 01001, RPERS 2018.

[2] Muzylev E.L., Uspenskii A.B., Startseva Z.P., Volkova E.V. et al. Remote determination of the underlying surface characteristics according to the data of scanning radiometers of the NOAA and EOS/TERRA satellites when modeling vertical moisture and heat fluxes from river catchments // Modern problems of Earth remote sensing from the space. 2007. Vol. 4. No. 2. P. 256-268.

[3] Brezhnev R.V., Maglinets Yu.A. Information support technique for solving agricultural land monitoring tasks based on Earth remote sensing data // Journal of SFU. Technocs and Technologies. 2017. Vol. 10. No. 6. P. 819-827.

[4] Weng Q., Lu D., Schubring J. Estimation of land surface temperature-vegetation abundance relationship for urban heat island studies // Remote Sensing of Environment. N.Y.: Elsevier Science Inc., 2004. Vol. 89. P. 467-483.

[5] Krapez J.-C., Olioso A., Coudert B. Comparison of three methods based on the TemperatureNDVI diagram for soil moisture characterization // SPIE Conference Remote Sensing for Agriculture, Ecosystems, and Hydrology XI. Berlin, 2009. Vol. 7472. 